\title{
A note on the Wang-Zhang and Schwarz inequalities
}

\author{
Sever S. Dragomir
}

\begin{abstract}
In this note we show that the Wang-Zhang inequality can be naturally applied to obtain an elegant reverse for the classical Schwarz inequality in complex inner product spaces.
\end{abstract}

Mathematics Subject Classification (2010): 46C05, 26D15.

Keywords: Schwarz inequality, inner products, inequalities for sums.

\section{Introduction}

Let $(H,\langle\cdot, \cdot\rangle)$ be a complex inner product space and $x, y \in H$ two nonzero vectors. One can define the angle between the vectors $x, y$ either by

$$
\Phi_{x, y}=\arccos \left(\frac{\operatorname{Re}\langle x, y\rangle}{\|x\|\|y\|}\right) \text { or by } \Psi_{x, y}=\arccos \left(\frac{|\langle x, y\rangle|}{\|x\|\|y\|}\right) .
$$

The function $\Psi_{x, y}$ is a natural metric on complex projective space [6]. vectors

In 1969 M. K. Kreln [5] obtained the following inequality for angles between two

$$
\Phi_{x, y} \leq \Phi_{x, z}+\Phi_{z, y}
$$

for any $x, y, z \in H \backslash\{0\}$.

By using the representation

$$
\Psi_{x, y}=\inf _{\alpha, \beta \in \mathbb{C} \backslash\{0\}} \Phi_{\alpha x, \beta y}=\inf _{\alpha \in \mathbb{C} \backslash\{0\}} \Phi_{\alpha x, y}=\inf _{\beta \in \mathbb{C} \backslash\{0\}} \Phi_{x, \beta y}
$$

and Krĕn's inequality (1.1), M. Lin [6] has shown recently that the following triangle inequality is also valid

$$
\Psi_{x, y} \leq \Psi_{x, z}+\Psi_{z, y}
$$

for any $x, y, z \in H \backslash\{0\}$. 
The following inequality has been obtained by Wang and Zhang in [9] (see also $[11$, p. 195])

$$
\sqrt{1-\frac{|\langle x, y\rangle|^{2}}{\|x\|^{2}\|y\|^{2}}} \leq \sqrt{1-\frac{|\langle x, z\rangle|^{2}}{\|x\|^{2}\|z\|^{2}}}+\sqrt{1-\frac{|\langle y, z\rangle|^{2}}{\|y\|^{2}\|z\|^{2}}}
$$

for any $x, y, z \in H \backslash\{0\}$. Using the above notations it can be written as [6]

$$
\sin \Psi_{x, y} \leq \sin \Psi_{x, z}+\sin \Psi_{z, y}
$$

for any $x, y, z \in H \backslash\{0\}$. It also provides another triangle type inequality complementing the Kreı̀n and Lin inequalities above.

In this note we show that the Wang-Zhang inequality can be naturally applied to obtain an elegant reverse for the classical Schwarz inequality in complex inner product spaces.

\section{Reverse of Schwarz inequality}

In the sequel we assume that $(H,\langle\cdot, \cdot\rangle)$ is a complex inner product space. The inequality

$$
|\langle x, y\rangle|^{2} \leq\|x\|^{2}\|y\|^{2} \text { for } x, y \in H
$$

is well know in the literature as the Schwarz inequality. The equality holds in (2.1) iff $x$ and $y$ are linearly dependent.

Theorem 2.1. Let $x, y, z \in H$ with $\|z\|=1$ and $\alpha, \beta \in \mathbb{C}, r, s>0$ such that

$$
\|x-\alpha z\| \leq r \text { and }\|y-\beta z\| \leq s .
$$

Then

$$
(0 \leq)\|x\|^{2}\|y\|^{2}-|\langle x, y\rangle|^{2} \leq(r\|y\|+s\|x\|)^{2} .
$$

Proof. If we multiply (1.4) by $\|x\|\|y\|\|z\|>0$, then we get

$$
\begin{aligned}
& \|z\| \sqrt{\|x\|^{2}\|y\|^{2}-|\langle x, y\rangle|^{2}} \\
\leq & \|y\| \sqrt{\|x\|^{2}\|z\|^{2}-|\langle x, z\rangle|^{2}}+\|x\| \sqrt{\|y\|^{2}\|z\|^{2}-|\langle y, z\rangle|^{2}}
\end{aligned}
$$

for any $x, y, z \in H \backslash\{0\}$.

We observe that, if either $x=0$ or $y=0$, then the inequality (2.4) reduces to an equality.

Let $z \in H$ with $\|z\|=1$, and since (see for instance [2, Lemma 2.4])

$$
\|x\|^{2}-|\langle x, z\rangle|^{2}=\inf _{\lambda \in \mathbb{C}}\|x-\lambda z\|^{2} \text { and }\|y\|^{2}-|\langle y, z\rangle|^{2}=\inf _{\mu \in \mathbb{C}}\|y-\mu z\|^{2}
$$

then by (2.4) we have

$$
\sqrt{\|x\|^{2}\|y\|^{2}-|\langle x, y\rangle|^{2}} \leq\|y\| \inf _{\lambda \in \mathbb{C}}\|x-\lambda z\|+\|x\| \inf _{\mu \in \mathbb{C}}\|y-\mu z\|,
$$

for any $x, y, z \in H$ with $\|z\|=1$. 
Since, by $(2.2)$

$$
\inf _{\lambda \in \mathbb{C}}\|x-\lambda z\| \leq\|x-\alpha z\| \leq r \text { and } \inf _{\mu \in \mathbb{C}}\|y-\mu z\| \leq\|y-\beta z\| \leq s,
$$

then by (2.5) we obtain the desired result (2.3).

Corollary 2.2. Let $x, y, z \in H$ with $\|z\|=1$ and $\lambda, \Lambda, \gamma, \Gamma \in \mathbb{C}$ with $\lambda \neq \Lambda, \gamma \neq \Gamma$ and such that either

$$
\operatorname{Re}\langle\Lambda z-x, x-\lambda z\rangle \geq 0 \text { and } \operatorname{Re}\langle\Gamma z-y, y-\gamma z\rangle \geq 0
$$

or, equivalently

$$
\left\|x-\frac{\lambda+\Lambda}{2} z\right\| \leq \frac{1}{2}|\Lambda-\lambda| \text { and }\left\|y-\frac{\gamma+\Gamma}{2} z\right\| \leq \frac{1}{2}|\Gamma-\gamma|
$$

are valid. Then

$$
(0 \leq)\|x\|^{2}\|y\|^{2}-|\langle x, y\rangle|^{2} \leq \frac{1}{4}(|\Lambda-\lambda|\|y\|+|\Gamma-\gamma|\|x\|)^{2} .
$$

Proof. Follows by Theorem 2.1 on observing that

$$
\operatorname{Re}\langle\Delta e-u, u-\delta e\rangle=\frac{1}{4}|\Delta-\delta|^{2}-\left\|u-\frac{\delta+\Delta}{2} e\right\|^{2}
$$

for any $\delta, \Delta \in \mathbb{C}$ with $\delta \neq \Delta$ and $u, e \in H$ with $\|e\|=1$.

We give an example for $n$-tuples of complex numbers.

Let $x=\left(x_{1}, \ldots, x_{n}\right), y=\left(y_{1}, \ldots, y_{n}\right)$ and $z=\left(z_{1}, \ldots, z_{n}\right)$ be $n$-tuples of complex numbers, $p=\left(p_{1}, \ldots, p_{n}\right)$ a probability distribution, i.e. $p_{i}>0 i \in\{1, \ldots, n\}$ and $\sum_{i=1}^{n} p_{i}=1$, with $\sum_{i=1}^{n} p_{i}\left|z_{i}\right|^{2}=1$ and $\lambda, \Lambda, \gamma, \Gamma \in \mathbb{C}$ with $\lambda \neq \Lambda, \gamma \neq \Gamma$ and such that

$$
\operatorname{Re}\left[\left(\Lambda z_{i}-x_{i}\right)\left(\bar{x}_{i}-\bar{\lambda} \bar{z}_{i}\right)\right] \geq 0 \text { and } \operatorname{Re}\left[\left(\Gamma z_{i}-\bar{y}_{i}\right)\left(\bar{y}_{i}-\bar{\gamma}_{i}\right)\right] \geq 0
$$

or, equivalently

$$
\left|x_{i}-\frac{\lambda+\Lambda}{2} z_{i}\right| \leq \frac{1}{2}|\Lambda-\lambda| \text { and }\left|y_{i}-\frac{\gamma+\Gamma}{2} z_{i}\right| \leq \frac{1}{2}|\Gamma-\gamma|
$$

for any $i \in\{1, \ldots, n\}$. Then

$$
\sum_{i=1}^{n} p_{i} \operatorname{Re}\left[\left(\Lambda z_{i}-x_{i}\right)\left(\bar{x}_{i}-\bar{\lambda} \bar{z}_{i}\right)\right] \geq 0 \text { and } \sum_{i=1}^{n} p_{i} \operatorname{Re}\left[\left(\Gamma z_{i}-\bar{y}_{i}\right)\left(\bar{y}_{i}-\overline{\gamma z_{i}}\right)\right] \geq 0
$$

and by applying Corollary 2.2 for the inner product $\langle\cdot, \cdot\rangle_{p}: \mathbb{C}^{n} \times \mathbb{C}^{n} \rightarrow \mathbb{C}$ with

$$
\langle x, y\rangle_{p}=\sum_{i=1}^{n} p_{i} x_{i} \bar{y}_{i}
$$


we have

$$
\begin{aligned}
0 & \leq \sum_{i=1}^{n} p_{i}\left|x_{i}\right|^{2} \sum_{i=1}^{n} p_{i}\left|y_{i}\right|^{2}-\left|\sum_{i=1}^{n} p_{i} x_{i} \bar{y}_{i}\right|^{2} \\
& \leq \frac{1}{4}\left[|\Lambda-\lambda|\left(\sum_{i=1}^{n} p_{i}\left|y_{i}\right|^{2}\right)^{1 / 2}+|\Gamma-\gamma|\left(\sum_{i=1}^{n} p_{i}\left|x_{i}\right|^{2}\right)^{1 / 2}\right]^{2} .
\end{aligned}
$$

If $0<a \leq a_{i} \leq A<\infty$ and $0<b \leq b_{i} \leq B<\infty$ for any $i \in\{1, \ldots, n\}$ then by (2.8) we have for any $p=\left(p_{1}, \ldots, p_{n}\right)$ a probability distribution that

$$
\begin{aligned}
0 & \leq \sum_{i=1}^{n} p_{i} a_{i}^{2} \sum_{i=1}^{n} p_{i} b_{i}^{2}-\left(\sum_{i=1}^{n} p_{i} a_{i} b_{i}\right)^{2} \\
& \leq \frac{1}{4}\left[(A-a)\left(\sum_{i=1}^{n} p_{i} b_{i}^{2}\right)^{1 / 2}+(B-b)\left(\sum_{i=1}^{n} p_{i} a_{i}^{2}\right)^{1 / 2}\right]^{2} .
\end{aligned}
$$

The interested reader may compare this new result with the classical reverses of Schwarz inequality obtained by Diaz and Metcalf [1], Ozeki [4], G. Pólya and G. Szegö [7], Shisha and Mond [8] and Cassels [10].

For other reverses of Schwarz inequality in complex inner product spaces see the monograph [3] and the references therein.

Acknowledgement. The author would like to thank the anonymous referee for some valuable suggestions that have been implemented in the final version of the manuscript.

\section{References}

[1] Diaz, J.B., Metcalf, F.T., Stronger forms of a class of inequalities of G. Pólya-G. Szegö and L.V. Kantorovich, Bull. Amer. Math. Soc., 69(1963), 415-418.

[2] Dragomir, S.S., Some Grüss type inequalities in inner product spaces, J. Inequal. Pure Appl. Math., 4(2003), No. 2, Article 42, 10 pp.

[3] Dragomir, S.S., Advances in Inequalities of the Schwarz, Grüss and Bessel Type in Inner Product Spaces, Nova Science Publishers, Inc., Hauppauge, NY, 2005. viii+249 pp.

[4] Izumino, S., Pečarić, J., A weighted version of Ozeki's inequality, Sci. Math. Japonicae, 56(2002), no. 3, 511-526.

[5] Kreı̆n, M.K., Angular localization of the spectrum of a multiplicative integral in a Hilbert space, Funct. Anal. Appl., 3(1969), 89-90.

[6] Lin, M., Remarks on Krĕ̌n's inequality, The Math. Intelligencer, 34(2012), no. 1, 3-4.

[7] Pólya, G., Szegö, G., Problems and Theorems in Analysis, Volume 1: Series, Integral Calculus, Theory of Functions (in English), translated from german by D. Aeppli, corrected printing of the revised translation of the fourth German edition, Springer Verlag, New York, 1972.

[8] Shisha, O., Mond, B., Bounds on Differences of Means, Inequalities, Academic Press Inc., New York, 1967, pp. 293-308. 
[9] Wang, B., Zhang, F., A trace inequality for unitary matrices, Amer. Math. Monthly, 101(1994), 453-455.

[10] Watson, G.S., Alpargu, G., Styan, G.P.H., Some comments on six inequalities associated with the inefficiency of ordinary least squares with one regressor, Linear Algebra and its Appl., 264(1997), 13-54.

[11] Zhang, F., Matrix Theory: Basic Results and Techniques, Springer-Verlag, New York, 2011

Sever S. Dragomir

Mathematics, College of Engineering \& Science

Victoria University, PO Box 14428

Melbourne City, MC 8001, Australia

sever.dragomir@vu.edu.au

http://rgmia.org/dragomir

School of Computer Science \& Applied Mathematics

University of the Witwatersrand, Private Bag 3

Johannesburg 2050, South Africa

e-mail: sever.dragomir@vu.edu.au 